\title{
Monitoring Conformational Changes in an Enzyme Conversion Inhibitor Using Pure Shift Exchange NMR Spectroscopy
}

\author{
G. Aloui, ${ }^{[a, b]}$ S. Bouabdallah, ${ }^{[b]}$ J.P. Baltaze, ${ }^{[a]}$ J. E. H. Pucheta, ${ }^{[c]}$ S. Touil, ${ }^{[b]}$ J. Farjon, ${ }^{[d]}$ and \\ N. Giraud*[a, e]
}

We report the acquisition of 2D NMR EXSY spectra with ultrahigh resolution, which allows for probing the slow conformational exchange process in a pharmaceutical compound. The resolution enhancement is achieved by implementing interferogram based PSYCHE homonuclear decoupling to generate a pure shift proton spectrum along the direct domain of the resulting data. The performance of this pure shift EXSY pulse sequence is compared to the standard experiment recorded under identical conditions. It is found that although being less sensitive and requiring a longer acquisition time, the quality of pure shift spectra allows for extracting exchange rates values that are coherent with the ones determined by standard approach, on a temperature range that demonstrates the robustness of the chosen homonuclear decoupling method. The resolution enhancement provided by the simplification of proton line shape allows for probing a higher number of proton sites whose analysis would have been biased using a standard method. These results open the way to a thorough and accurate study of chemical exchange processes based on a multi-site analysis of 2D pure shift EXSY spectra

\section{Introduction}

Trandolapril is a long-lasting angiotensin converting enzyme ACE inhibitor that plays a major role in the regulation of blood pressure. ${ }^{[1]}$ An analysis of this commercial prodrug in $\mathrm{CDCl}_{3}$ by nuclear magnetic resonance (NMR) revealed that it has two conformations, and that the interconversion between the two conformers involves a rotation about the amide bound (Figure 1). The geometry of each conformer was determined by DFT calculation, with an energy difference of about $6 \mathrm{~kJ} \mathrm{~mol}^{-1}$ between the cis (preferred) and trans conformer. ${ }^{[2]}$ Since the

[a] G. Aloui, J. P. Baltaze, N. Giraud

Université Paris Saclay

Institut de Chimie Moléculaire et des Matériaux d'Orsay

Equipe RMN en Milieu Orienté

UMR CNRS-UPS 8182, 91405 Orsay, France

[b] G. Aloui, S. Bouabdallah, S. Touil

Laboratory of Hetero-Organic Compounds and Nanostructured Materials University of Carthage, Faculty of Sciences of Bizerte 7021 Jarzouna, Tunisia

[c] J. E. H. Pucheta

Consejo Nacional de Ciencia y Tecnología - Laboratorio Nacional de Investigación y Servicio Agroalimentario y Forestal, Universidad Autónoma Chapingo

Km. 38.5 Carretera México-Texcoco, Chapingo 56230, Estado de México, México

[d] J. Farjon

CEISAM UMR CNRS 6230

Faculté des Sciences et Techniques

2 rue de la Houssinière, BP 92208, 44322 Nantes cedex 3 France

[e] $N$. Giraud

Laboratory of Pharmacological and Toxicological Chemistry and Biochemistry

Université Paris Descartes, Sorbonne Paris Cité

45 rue des Saints Pères, 75006 Paris, France

E-mail: nicolas.giraud@parisdescartes.fr

Supporting information for this article is available on the WWW under https://doi.org/10.1002/cphc.201900244

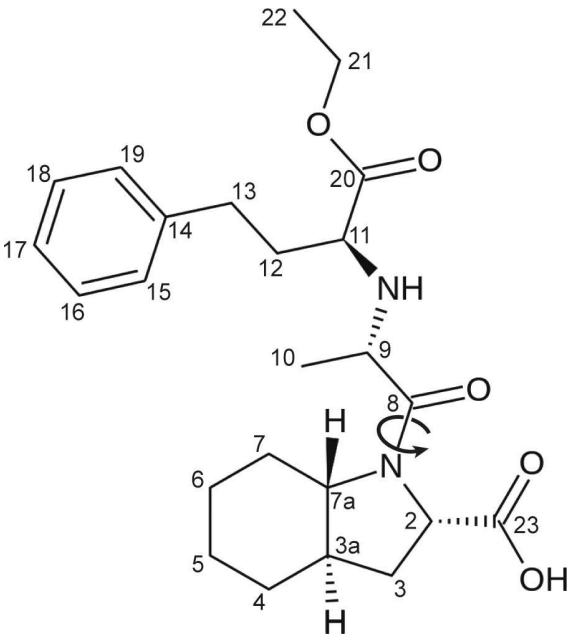

Figure 1. The structure and labeling of Trandolapril. The arrow indicates the main chemical bond involved in the conformational equilibrium as described elsewhere. ${ }^{[2]}$

existence of different conformers of pharmaceutical products can affect their biological activity before and during their metabolisation, characterizing each conformer and determining the time scale of the interconversion process has become a major analytical issue in pharmaceutical industry. In this context, nuclear magnetic resonance (NMR) spectroscopy has proved to be a tool of choice for monitoring chemical, and more specifically conformational exchange. A wide range of methods has long been developed, which provide access to kinetic and thermodynamic features of the involved equilibrium, depending on its time scale and its influence on the resulting NMR spectrum. ${ }^{[3-6]}$ 
For Trandolapril, in the temperature range corresponding to physiological conditions $\left(20-40^{\circ} \mathrm{C}\right)$, the regime of the conformational exchange is slow, leading to the observation of doubled ${ }^{1} \mathrm{H}$ and ${ }^{13} \mathrm{C}$ resonances, on spectrometers operating at proton resonance frequencies in the range $400-600 \mathrm{MHz} .{ }^{[2]}$

When the exchange rate is on the same time scale as the longitudinal relaxation of the probed nucleus, exchange spectroscopy (EXSY) provides a convenient way of identifying and analyzing doubled resonances, while taking benefit of the dispersion of correlation peaks across multi-dimensional spectra. EXSY experiments are among the earlier multi-dimensional experiments. ${ }^{[7]}$ They allow for probing physical processes in the $10-2000 \mathrm{~ms}$ time window. ${ }^{[8-13]}$ These processes include slow conformational changes such as domain movement, ${ }^{[14,15]}$ ligand binding ${ }^{[16,17]}$ and release, ${ }^{[18]}$ topological interconversion of secondary structure ${ }^{[19,20]}$ and cis-trans isomerization, ${ }^{[21]}$ all of which may affect catalytic turnover rates in enzymes. ${ }^{[12,22,23]} \mathrm{A}$ problem that is however often encountered in such approach is the spectral resolution that can be achieved. Indeed, the two species that are interconverting constitute two different spin systems, and the resulting spectrum is the sum of two different sets of signals that are at least partially overlapping.

Recently, Thiele et al. have reported an interferogram-based Zangger-Sterk (ZS) pure shift NOESY experiment that allows for recording 2D NOESY spectra, the correlations of which show a singlet structure in the reconstructed "direct" spectral dimension (F2). ${ }^{[24]}$ This simplification of the observed lineshape allows for limiting signal overlap, which contributes to increase significantly the resolution. More generally, they have shown that it is possible to use interferogram-based Zangger-Sterk ${ }^{1} \mathrm{H}$ pure shift NMR experiments to determine NOE-based interatomic distances, with a good agreement with structural model for small organic compounds. It was notably shown that the quality of the resulting data allows for quantifying nuclear Overhauser effect (nOe) and extracting nOe-derived distance restraints with a good accuracy on small organic compounds. Thiele et al also showed that a post-processing of the data can be applied to reduce the artifacts that are due to the interferogram based acquisition scheme.

In this paper, we propose to make use of this approach to record pure shift EXSY spectra and improve the quality of the analysis of chemical exchange. To that end, we first evaluate the robustness of the chosen interferogram-based broadband homonuclear decoupling ${ }^{[25-29]}$ acquisition scheme, in the particular case of a sample undergoing conformational exchange. We report the implementation of a slight evolution of the pulse sequence introduced by Thiele et al. in which we use the PSYCHE method ${ }^{[30,31]}$ for performing homonuclear decoupling. We compare the resulting EXSY spectra with the analogous $2 \mathrm{D}$ maps acquired without homonuclear decoupling, and we evaluate the quality of the kinetic and thermodynamic parameters that can be extracted from the analysis of the correlations measured on pure shift EXSY datasets.

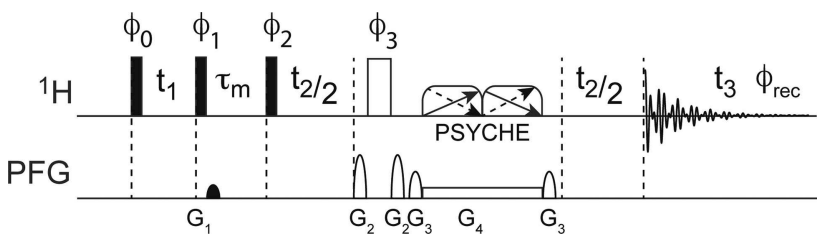

Figure 2. Pulse sequence for 2D F2-PSYCHE-EXSY experiment. The narrow black rectangles and the wide white rectangles are $90^{\circ}$ and $180^{\circ}$ RF pulses, respectively. The trapezoids with diagonal are homonuclear decoupling block PSYCHE. The pulse phases are $\phi_{0}=x, \phi_{1}=x-x, \phi_{2}=x x-x-x, \phi_{3}=x$ $x \mathrm{x} x$ y y $\mathrm{y}, \phi_{\text {rec }}=\mathrm{x}-\mathrm{x}-\mathrm{x} \mathrm{x}-\mathrm{x} \mathrm{x} \mathrm{x}-\mathrm{x}$.

\section{Experimental Section}

Experiments were carried out on $15 \mathrm{mg}$ of Trandolapril dissolved in $600 \% \mathrm{~L}$ of deuterated methanol. NMR spectra were acquired on a Bruker AVANCE II NMR spectrometer operating at $14.1 \mathrm{~T}$, equipped with a $5-\mathrm{mm}^{2} \mathrm{H}-{ }^{1} \mathrm{H}$ cryoprobe with $z$ field gradient coil.

\section{Pure Shift ${ }^{1} \mathrm{H}$ NMR}

Pure shift 1D spectra (Figure 2) were recorded using the pulse sequence implemented with the PSYCHE method and published elsewhere. ${ }^{[32,33]}$ The duration of the $\pi / 2$ pulse was $20.2 \mu$ s for ${ }^{1} \mathrm{H}$. The chirp pulses were centered at $5.4 \mathrm{ppm}$, and their durations were set to $15 \mathrm{~ms}$ each, to cover about $10 \mathrm{kHz}$ with an amplitude of $33.7 \mathrm{~Hz}$ and an experimentally optimized angle $\beta$ of $25^{\circ}$, which is a compromise between sensitivity and artifacts generated by the PSYCHE element. The pulsed field gradients used for the selection of coherence transfer pathways were applied with amplitudes of $40.8 \mathrm{G} \mathrm{cm}^{-1}$, and $26.0 \mathrm{G} \mathrm{cm}^{-1}$ respectively and a duration of $1 \mathrm{~ms}$ each. The amplitude of the gradient pulse aligned with the center of the pulse element consisting of the two chirp pulses was experimentally optimized to $0.53 \mathrm{G} \mathrm{cm}^{-1}$. All gradient pulses were followed by a recovery delay of $150 \mu \mathrm{s}$. The free induction decay was reconstructed from the acquisition of a series of 32 chunks of $20 \mathrm{~ms}$ each. The spectral windows in $F_{1}$ and $F_{2}$ were set to $33 \mathrm{~Hz}$ and $4500 \mathrm{~Hz}$ respectively. For each chunk, a free induction decay of $16 \mathrm{k}$ points was acquired, with 4 scans and recycle delays between scans of $1.5 \mathrm{~s}$. The resulting pseudo-2D data were processed using the pshift macro to generate the 1D signal.

\section{Standard EXSY Experiments}

The standard NOESY pulse sequence was used to record EXSY 2D spectra. ${ }^{[7,34]}$ For each of the 512 increments in $t_{1}$, a free induction decay of 18028 points was acquired, with 2 scans. A 3 s recycle delay between scans was set, so that (i) large series of 2D EXSY spectra can be acquired within an amount of time that is compatible with a routine analytical protocol, and (ii) the relaxation properties in this compound allow for restoring $>95 \%$ of longitudinal magnetization at thermal equilibrium. Few proton sites for which this latter condition was not satisfied were excluded from the analysis of the conformational exchange, as it is the case for instance for $\mathrm{H}^{11}$. The spectral window was set to $7.5 \mathrm{ppm}$. Data were processed by using zero-filling up to 1024 points in $t_{1}$, and 8192 points in $t_{2}$, apodization of $0.3 \mathrm{~Hz}$ and automatic baseline correction in both dimensions. The evolution of EXSY spectra was monitored for a series of six mixing times $(200,400,600,800,1000$, $1200 \mathrm{~ms}$ ) for standard and pure shift studies. 

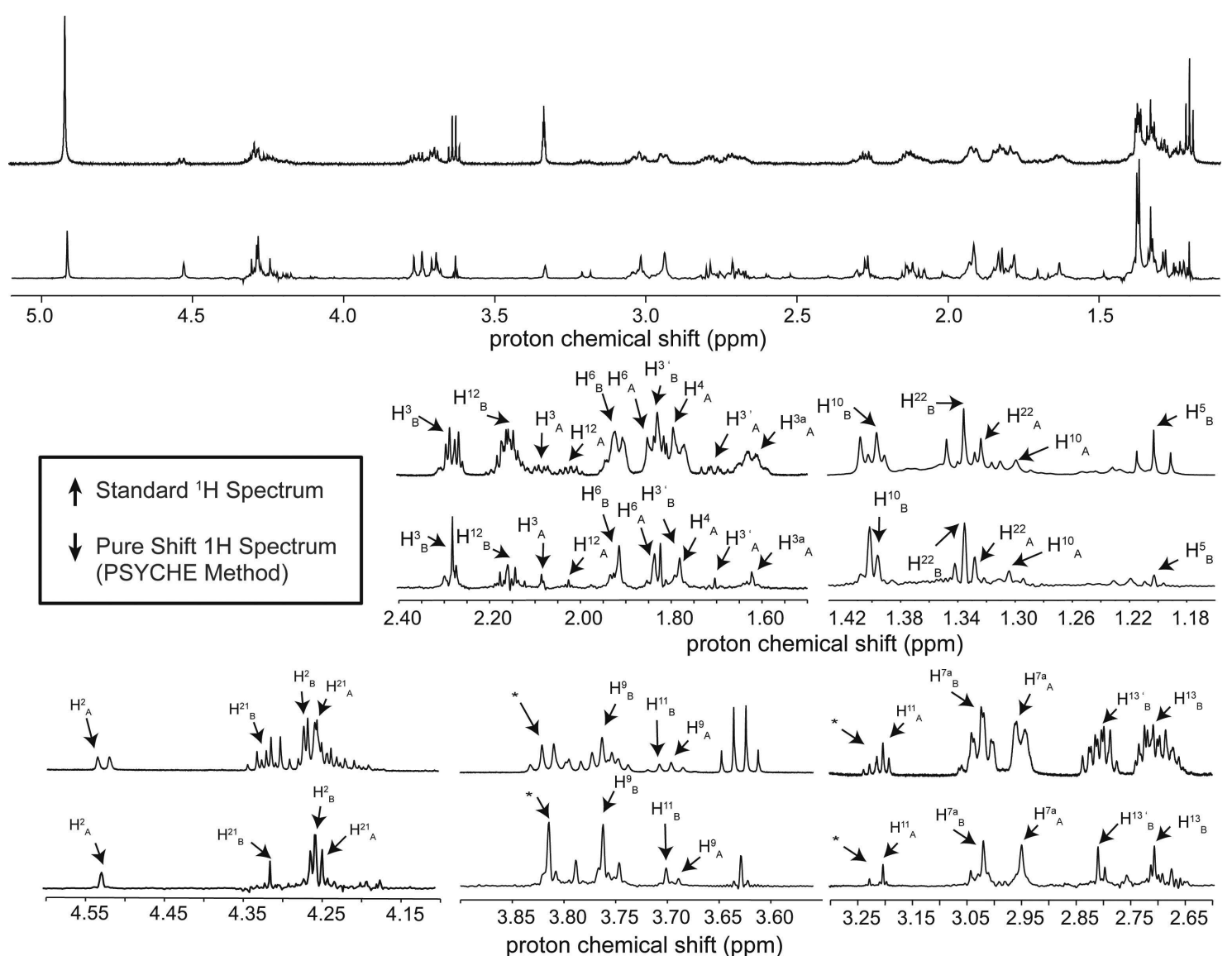

Figure 3. 1D 1H NMR Spectra recorded on Trandolapril dissolved in MeOD at $300 \mathrm{~K}$, (top) using a standard acquisition scheme, (bottom) using a pseudo 2D pulse sequence implementing PSYCHE broadband homonuclear decoupling scheme.

\section{F2-PSYCHE-EXSY Experiments}

The pure-shift NOESY pulse sequence introduced by Thiele et al. was slightly modified by replacing the ZS element with a PSYCHE element (Figure 2). Pulsed field gradients amplitudes used were $\mathrm{G}_{1}=5.3 \mathrm{Gcm}^{-1}, \mathrm{G}_{2}=40.8 \mathrm{Gcm}^{-1}, \mathrm{G}_{3}=26 \mathrm{Gcm}^{-1}, \mathrm{G}_{4}=0.5 \mathrm{Gcm}^{-1}$, and a duration of $1 \mathrm{~ms}$ each (except for $\mathrm{G} 4$ whose duration is that of the PSYCHE element). Each free induction decay was reconstructed from the acquisition of 14 chunks of 30.2 ms each. For each chunk, a free induction decay of 1024 points was acquired with 4 scans and a recycle delay between scans of $1 \mathrm{~s}$.

The spectral window was set to $7.5 \mathrm{ppm}$. The data chunks were concatenated to produce an FID of 437.8 ms with 3944 complex data points and zero-filled to 8192 complex data points. An exponential apodization ( $0.3 \mathrm{~Hz}$ line broadening) was applied.

Automatic baseline correction was applied to the spectrum before manual integration of the peaks.

\section{Results and Discussion}

\subsection{Pure Shift Spectroscopy}

Performing pure shift NMR on molecules undergoing chemical exchange can be challenging. In the case of labile protons in the fast exchange regime with either the solvent or other solute molecules, it is often observed that their signal disappears or is at least strongly affected by the application of the gradient encoded pulse schemes that are used for triggering pure shift or J-edited spin evolutions. This is the case for instance for ZSbased methods. An alternative consists in using a solvent that cancels or slows down the kinetics of the chemical exchange to a point where protons such as those from hydroxy or amine groups can be observed, and their residence time is long enough to act on the evolution of their scalar couplings with neighboring protons. ${ }^{[35]}$

Here we have chosen to evaluate the robustness of the PSYCHE method for recording pure shift ${ }^{1} \mathrm{H}$ spectra of Trandolapril dissolved in methanol, under experimental conditions where the time scale of the interconversion process yields a set of doubled proton resonances. It should be reminded that the PSYCHE method can allow for a higher sensitivity than the ZS method and thus for acquiring datasets in a reduced amount of experimental time, sometimes at the cost of spectral artifacts, which needs to be evaluated in the context of the analysis of a mixture potentially containing a minor form. ${ }^{[36]}$

The standard and pure shift ${ }^{1} \mathrm{H}$ spectra recorded on the Trandolapril sample at $300 \mathrm{~K}$ are shown in Figure 3. As 
expected, we observe a simplification of the multiplets observed on the standard spectrum, and a clear separation of signals with close chemical shifts, as it is highlighted by the assignment indicated on magnified regions of interest.

For instance, the two resonances of proton $\mathrm{H}^{10}$ in each conformer A (trans) and B (cis) can be clearly resolved, whereas a signal overlap with $\mathrm{H}^{12}$ a was preventing their analysis on the standard spectrum. We also remark that the chosen pure shift scheme achieves a less efficient decoupling of protons resonating between 4.15 and 4.40 ppm, which arises from the presence of coupled protons with very close chemical shifts. This limit is common to several homonuclear decoupling methods, and this observation is thus coherent with what has already been discussed elsewhere. ${ }^{[33]}$ Few artifacts due to the chunk reconstruction, and other ones inherent to the PSYCHE method are also observed but are sufficiently weak to allow for implementing this experimental setting into a pure shift EXSY approach.

\subsection{Pure Shift Exchange Spectroscopy}

Following on these results, we have implemented a pure shift NOESY pulse sequence in which we have used the PSYCHE element (Figure 2). During the mixing time $\tau_{m}$, the evolution of the longitudinal magnetization that is created by the $\pi / 2$ pulse at the end of the first evolution delay $\left(t_{1}\right)$ is driven by spinlattice relaxation, cross relaxation, and chemical exchange. It is then converted to transverse magnetization by a $\pi / 2$ pulse. During the evolution delay $t_{2}$, homonuclear scalar couplings are refocused by the PSYCHE decoupling block, while the phase of transverse magnetization is only affected by its precession under chemical shift evolution. A chunk of data is then acquired during $t_{3}$, which is short enough to neglect the evolution of the scalar couplings. The free induction decay recorded for each $t_{1}$ increment is reconstructed from the series of chunks (corresponding to the delay $t_{3}$ ) acquired for incremented values of $t_{2}$ yielding a 2D dataset. ${ }^{[24]}$ The resulting F2-PSYCHE-EXSY experiment yields pseudo-3D datasets, which requires a longer experimental time to record than a standard 2D map.

The standard and pure shift EXSY spectra shown in Figure 4 were recorded using the same mixing time $\tau_{m}=1.2 \mathrm{~s}$. The same resolution was achieved, and identical processing was applied to the standard and pure shift spectra. We observe a very similar correlation pattern on each spectrum, notably for the positive cross peaks that highlight chemical exchange. The only regions where artifacts are observed correspond to coupled proton spins with close chemical shifts that were identified as being difficult to decouple for the PSYCHE methods on preliminary 1D pure shift analyses. Truncation artifacts are also observed for the correlations involving protons from the ethyl group $\mathrm{C}^{21} \mathrm{H}_{2}-\mathrm{C}^{22} \mathrm{H}_{3}$.

Figure 5 highlights the resolution enhancement that can be reached in the region around $3.5 \mathrm{ppm}$. On the standard EXSY spectrum, cross peaks arising from the chemical exchange involving proton nuclei $\mathrm{H}^{9}$ and $\mathrm{H}^{11}$ can be identified, but strong signal overlaps are observed. The same region taken from the a)

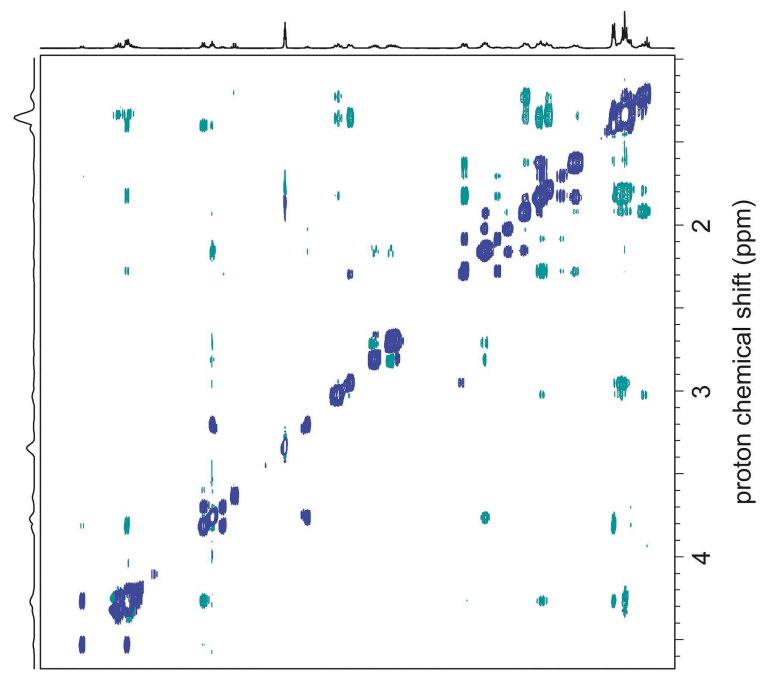

b)

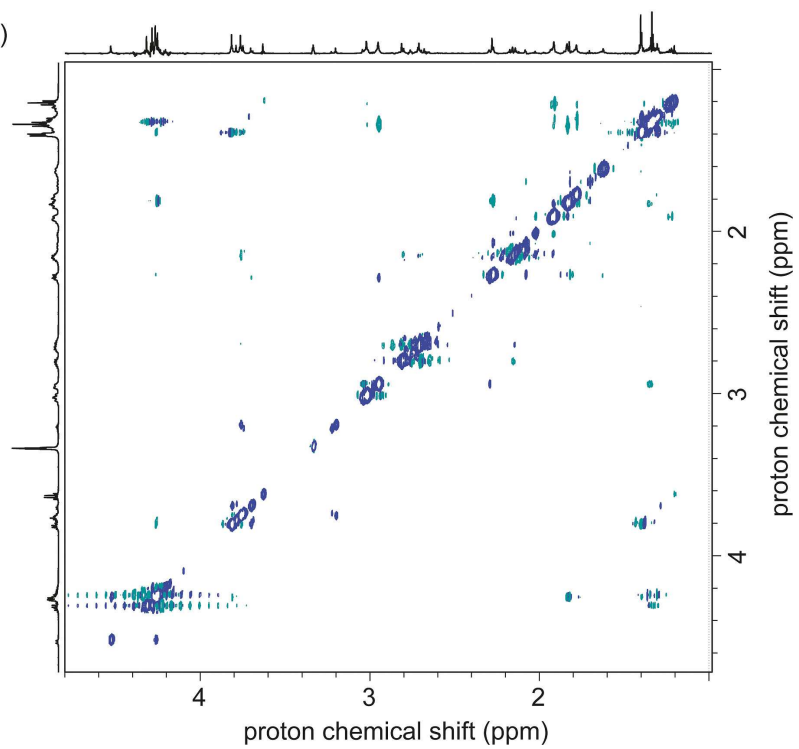

Figure 4. a) $2 \mathrm{D}$ standard EXSY spectrum recorded at $14.1 \mathrm{~T}$ in $1 \mathrm{~h} \mathrm{~b}$ ) $2 \mathrm{D}$ pure shift EXSY spectrum recorded in $14 \mathrm{~h}$ (for $\tau_{\mathrm{m}}=1.2 \mathrm{~s}$ ) at $14.1 \mathrm{~T}$ with the PSYCHE homonuclear decoupling method. The sample temperature was set to $290 \mathrm{~K}$. Grey lines correspond to negative cross peaks arising from crossrelaxation, and blue lines correspond to diagonal peaks, as well as positive cross peaks generated by chemical exchange.

pure shift EXSY spectrum allows for resolving these correlations, and thus separating exchange patterns of these two proton sites. An unexpected benefit of the resolution enhancement that can be achieved is the observation of weaker correlation patterns that were often hidden in the standard experiment. These weaker signals, which correspond to protons from a molecule that is also undergoing an exchange process as it is highlighted by the exchange cross peaks, are observed close to several doubled signals from Trandolapril. We could however not determine from the series of homonuclear and heteronuclear analyses that were carried out $\left({ }^{1} \mathrm{H}-{ }^{1} \mathrm{H}\right.$ COSY, TOCSY, ${ }^{1} \mathrm{H}-{ }^{13} \mathrm{C} \mathrm{HSQC} \mathrm{...)} \mathrm{if} \mathrm{this} \mathrm{sub-spin} \mathrm{system} \mathrm{could} \mathrm{fully} \mathrm{be} \mathrm{observed}$ on our data, which would have allowed us to conclude on a potential minor form arising from Trandolapril. Further analyses 

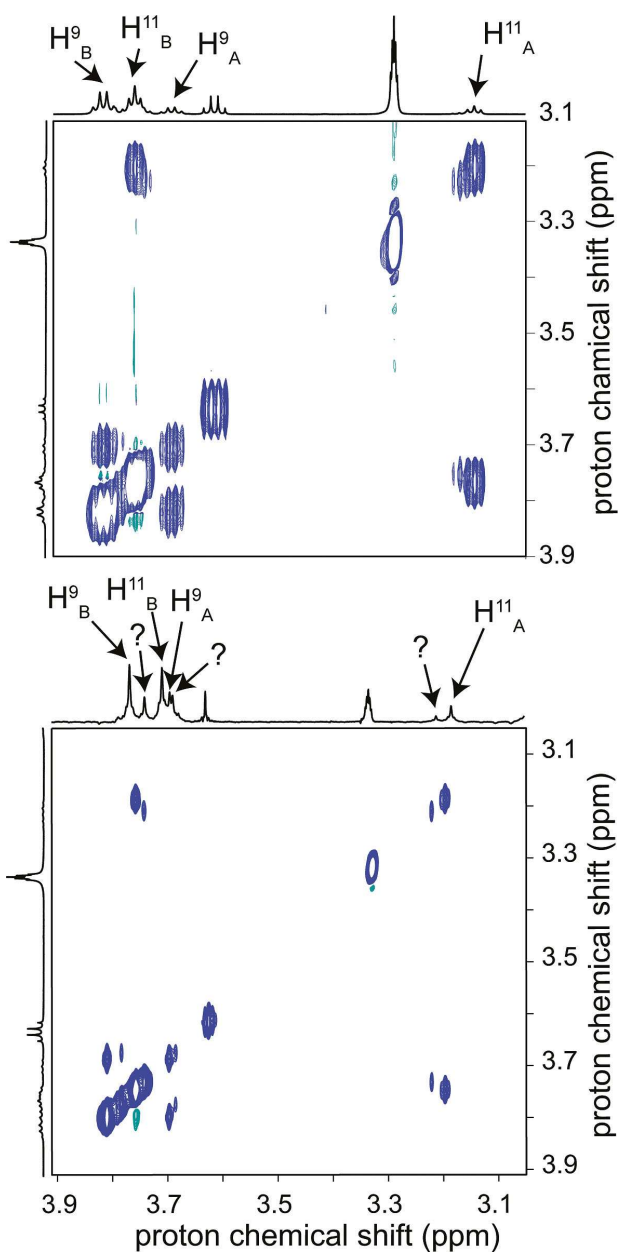

Figure 5. Selected region of standard and PSYCHE homodecoupled EXSY spectra shown in Figure 4. The correlations labeled with a ? correspond to an unidentified minor compound.

will be required to identify the compound corresponding to these signals that have not been described elsewhere.

Furthermore, it should be noted here that a similar resolution enhancement can be obtained by implementing the pure shift block in the middle of the indirect evolution delay $t_{1}$ of an EXSY pulse sequence, hence allowing for recording a $2 \mathrm{D}$ instead of a pseudo-3D dataset. We have thus recorded the corresponding F1-PSYCHE-EXSY experiment for the sake of comparison with the F2-PSYCHE-EXSY data presented in this work.

The pulse sequence, the resulting 2D EXSY spectrum (recorded with $\tau_{\mathrm{m}}=1.2 \mathrm{~s}$ ), and a comparison of selected correlations from standard EXSY, F2-PSYCHE-EXSY and F1PSYCHE-EXSY spectra are provided in ESI. Very similar resolution was achieved along the pure shift dimension in both experiments. We remark that the 2D F1-PSYCHE-EXSY was recorded in 8 hours, which is shorter than the 14 hours needed to record the F2-PSYCHE-EXSY spectrum with similar resolution, using the pseudo-3D acquisition scheme. The gain in experimental time is however not as substantial as it could be expected, due to the fact that a high number of increments needs to be recorded in the F1-PSYCHE-EXSY to reconstruct the indirect time domain and benefit from the resolution enhancement brought by the pure shift block. In the following, we are interested in evaluating the potential of the F2-PSYCHE-EXSY approach for the analysis of the conformational exchange process, and notably to describe the impact of the artefacts that arise from the reconstruction of the pure shift FID from a series of data chunks. Indeed, the results shown above suggest that the interconversion process between conformers $A$ and $B$ can be probed with a better accuracy, on a higher number of resolved proton sites using the F2-PSYCHE-EXSY approach. Following on from these results, we have studied the influence of the quality of these data on the determination of the kinetic and thermodynamic parameters of the conformational exchange. We have recorded a series of F2-PSYCHE-EXSY spectra with the mixing time $\tau_{\mathrm{m}}$ varied from 400 to $1200 \mathrm{~ms}$, using the standard and pure shift EXSY pulse sequences. For each series of $2 \mathrm{D}$ spectra, the volume of all the resolved correlation patterns was measured for each $\tau_{m}$ value. Figure 6 displays the resulting build-up curves that were obtained for $\mathrm{H}^{3}$ at 290 Kelvin. In order to evaluate the experimental uncertainty, we have repeated 6 times the acquisition of the standard and pure shift EXSY experiments with $\tau_{m}=1200 \mathrm{~ms}$. The standard deviation that was calculated over these 6 experiments for each cross-peak integration was assumed to be constant whatever the mixing time and was used as an error bar for the different points of the build-up curves. We observe that this experimental uncertainty
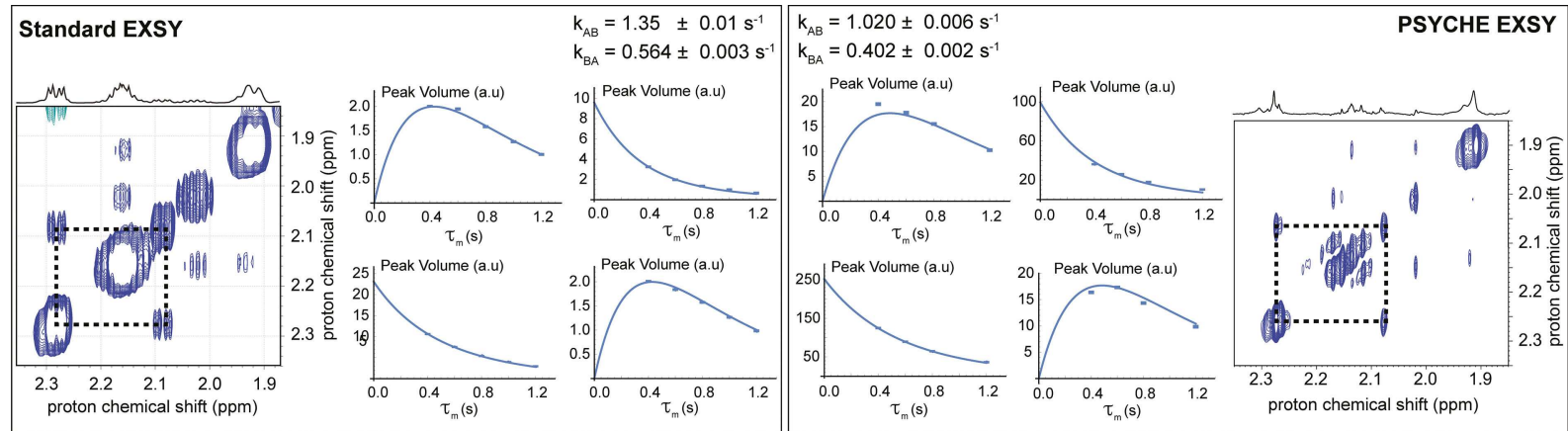

Figure 6. The regions of interest of the 2D standard and pure shift EXSY spectra corresponding to the correlation pattern assigned to $\mathrm{H}^{3}$ (2.1/2.3 ppm) are shown, together with the evolution of the signal integrations obtained by varying the mixing time $\tau_{\mathrm{m}}$ from $0.4 \mathrm{~s}$ to $1.2 \mathrm{~s}$ at $290 \mathrm{~K}$. 
is on average similar on pure shift and on standard data (the build-up curves that were obtained for other proton sites in Trandolapril are shown in the Supporting Information). The evolution of the diagonal and cross peaks arising from the exchange process between chemical species A and B can be modelled using the modified Bloch equation [Eq. (1)] $\square$ ok? $\square$ (at this step cross relaxation with other proton sites is neglected):

$\frac{d}{d t}\left[\begin{array}{c}M_{z}^{A} \\ M_{z}^{B}\end{array}\right]=\left[\begin{array}{cc}-\left(\frac{1}{T_{1}}+k_{A B}\right) & k_{B A} \\ k_{A B} & -\left(\frac{1}{T_{1}}+k_{B A}\right)\end{array}\right]\left[\begin{array}{c}M_{z}^{A}-M_{z}^{A}(e q) \\ M_{z}^{B}-M_{z}^{B}(e q)\end{array}\right]$

$M_{z}^{A}$ and $M_{z}^{B}$ are the nuclear magnetization in $A$ and $B, T_{1}$ is the longitudinal relaxation time that is assumed to be the same in $\mathrm{A}$ and $\mathrm{B}, k_{A B}$ and $k_{B A}$ are the exchange rates of the interconversion process, and $M_{z}^{A}(e q)$ and $M_{z}^{B}(e q)$ correspond to magnetizations at thermal equilibrium.

Diagonal and cross peaks integrations were fitted by the model curves that can be calculated through integration of equation (1) for different $\tau_{m}$ values. It was assumed that for each proton site the chosen homonuclear decoupling method had the same impact on relative signal integrals in each conformer, and that the relationship $M_{z}^{A}(e q) / M_{z}^{B}(e q)=k_{B A} / k_{A B}$ is always satisfied. This assumption seems to be reasonable in so far as relaxation, which is the main nuclear property that may induce different signal distortions during homonuclear decoupling blocks, is expected to be similar for a given proton in each conformer. In order to adjust the parameters of equation (1), the parameter space was sampled using a coarse grid search approach, and the reliability factor that was calculated to evaluate the distance between the model curve and the experimental points was locally minimized at each step of this protocol. The best fit corresponds to the absolute minimum of the reliability factor.

The blue curves plotted in Figure 6 for each dataset correspond to the best fit that was determined for $\mathrm{H}^{3}$ using this protocol. For the standard spectrum, it is apparent that both diagonal peaks overlap with other signals, whereas for the pure shift spectrum a better signal separation is observed. As a result, although both series of experimental points (standard and pure shift) can be fitted with the relaxation model corresponding to eq. 1 , it is expected that exchange rates determined on standard data are biased. The error bars are however similar, which underlines the good quality of the pure shift data. The accuracy on the determination of the parameters of the chemical exchange was estimated through a Monte Carlo based error analysis (the histograms showing the distributions of the exchange rates $k_{A B}, k_{B A}$ and $\mathrm{T}_{1}$ for $\mathrm{H}_{3}$ are shown in ESI). Slightly different mean values are obtained for $k_{A B}$ and $k_{B A}$, which is coherent with the small difference in the quality of the two datasets as described above.

We could probe the interconversion process between conformers A and B of Trandolapril on 11 different protons, using the standard and the pure shift EXSY approaches. The values of the exchange rates $k_{A B}$ and $k_{B A}$ are given for each site in Figure 7. Overall, we observe for each proton site that the

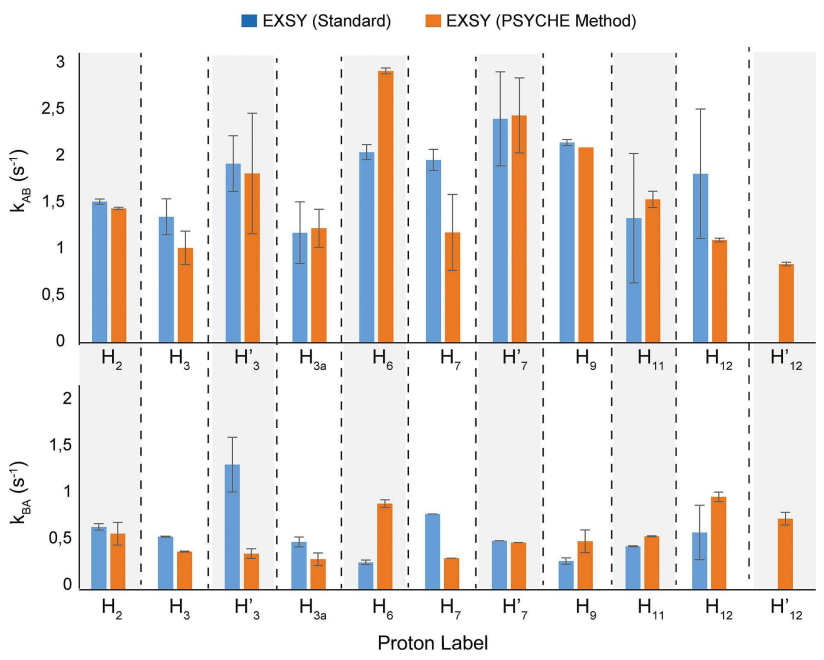

Figure 7. Diagrams of the exchange rates $k_{A B}$ and $k_{B A}$ extracted for the different protons sites in Trandolapril at $300 \mathrm{~K}$. Error bars correspond to twice the standard deviation of the distributions generated through a Monte Carlo based error analysis.

exchange rate values are similar in both datasets, except for a reduced number of protons.

For $\mathrm{H}_{3}^{\prime}, \mathrm{H}_{6}$, and $\mathrm{H}_{12}$, the differences between $k_{A B}$ (resp. $k_{B A}$ ) values determined by standard and pure shift approaches come from the fact that only a reduced number of correlations could be fitted, which is known to lead to a less accurate fitting of the chemical exchange model. For instance, it is apparent that the $k_{B A}$ value determined for $\mathrm{H}_{3}^{\prime}$ from standard EXSY spectra is significantly different from the other values determined on other proton sites, which is clearly correlated with the strong signals overlap involving these correlations. Furthermore, for $\mathrm{H}_{12}$, although a higher number of correlations could be fitted on standard (3 correlations) than on pure shift EXSY spectra ( 2 correlations), the resolution enhancement brought by homonuclear decoupling has led to a better accuracy of the exchange rates values determined by the latter approach, suggesting that these values are more reliable.

Moreover, for $\mathrm{H}_{7}$, pure shift data have allowed to fit all the correlations and thus to determine exchange rates values with a better accuracy. Similarly, for $\mathrm{H}^{\prime}{ }_{12}$, strong signal overlaps in standard EXSY data did not allow for fitting the correlations assigned to this proton site, whereas pure shift spectra provided an accurate fit (details about the quality of the analysis of all these datasets are summarized in sections 3 and 4 in ESI). Finally, if we account for the particular issues addressed above, we observe that the fluctuation of the determined exchange rates from one proton site to another is comparable for standard and pure shift datasets.

In particular, for correlation patterns that are sufficiently resolved on standard spectra to allow for an accurate extraction of the parameters of the interconversion process, we note that the results of the analyses made on pure shift and standard EXSY datasets are coherent. This suggests that the chosen homonuclear decoupling method does not induce any significant deviation in the measurement of the volume of the cross 

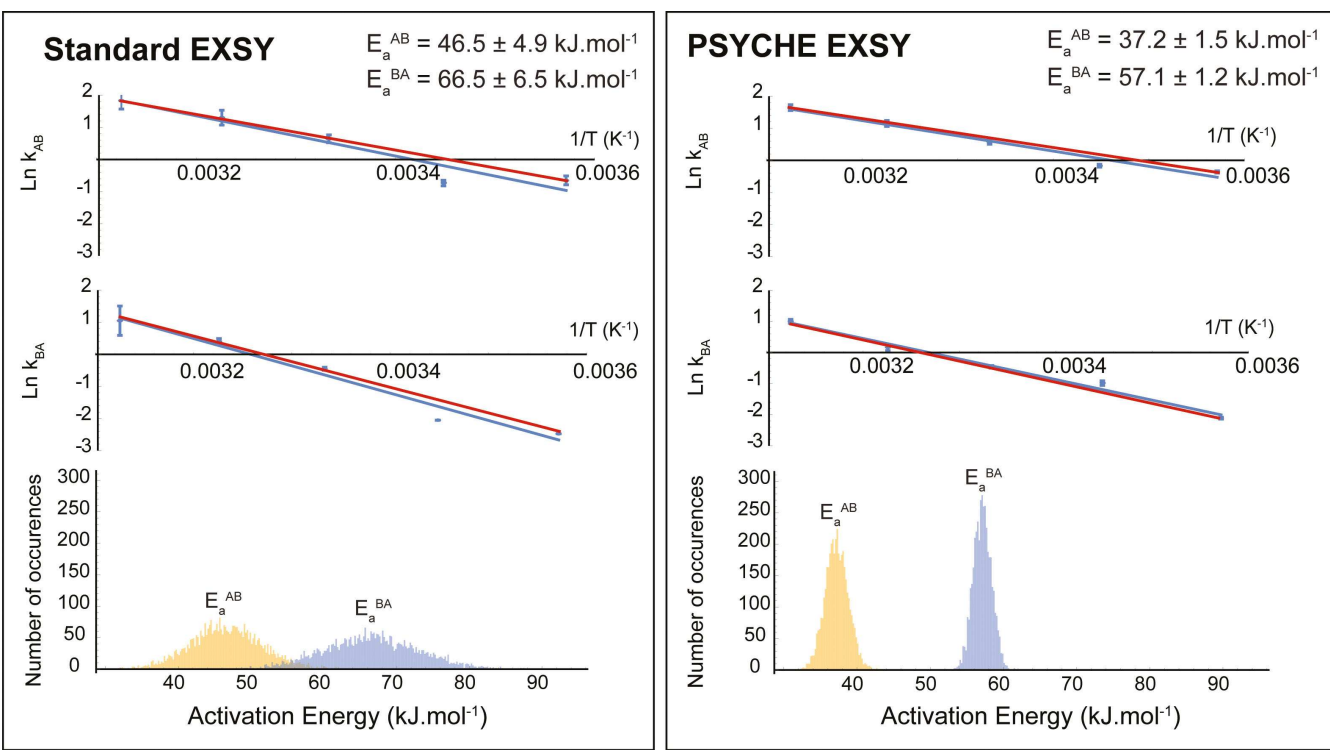

Figure 8. (Top) Arrhenius plots representing the evolution of $\operatorname{Ln}\left(k_{A B}\right)$ and $\operatorname{Ln}\left(k_{B A}\right)$ determined by varying the sample temperature, using the standard and pure shift EXSY approaches. (Bottom) The histograms of activation energies $E_{A B}$ and $E_{B A}$ that were obtained by Monte Carlo based error analysis.

peaks. We note however that the overall dispersion of $k_{A B}$ and $k_{B A}$ values is larger than the error bars that were evaluated by Monte Carlo simulations. This dispersion is coherent with the deviation that can be expected if the combined effect of (1) magnetization transfers by nuclear Overhauser effects and (2) the uncertainty on the measurement of correlations volumes, which is expected to have an impact on the measurement of weak correlations, are accounted for. A rough simulation of this contribution yields additional deviations in the range 10 to $20 \%$ for the determination of exchange rates values when build-up curves are fitted with a relaxation model that neglects nOe transfers. In the following, it will be assumed that the mean value of $k_{A B}$ 's and $k_{B A}$ 's calculated over all the proton sites in Trandolapril gives an acceptable estimate of the true values of these exchange rates.

An important feature of the exchange process is the activation energy that drives the interconversion between the exchanging species. This energy can be evaluated through the analysis of the temperature dependence of the exchange rates. For such an activated process, we remind that the relationship between $k_{A B}, k_{B A}$ and the corresponding activation energies $E_{A B}$ and $E_{B A}$ is given by the Arrhenius equation [Eq. (2)]:

$k_{A B / B A}=A_{A B / B A} \cdot e^{-E_{A B / B A} / k_{B} T}$

where $A_{A B / B A}$ is the pre-exponential factor, $k_{B}$ is the Boltzmann constant, and $T$ is the temperature. We have studied the influence of $T$ on pure shift EXSY spectra in order to determine if this experiment can be exploited to evaluate the activation energies of the interconversion process in Trandolapril. To that end, we have recorded the series of standard and pure shift EXSY spectra at 5 different temperatures ranging from 280 to $320 \mathrm{~K}$. For each temperature the same analysis as described above was performed to extract the mean values of $k_{A B}$ and $k_{B A}$ together with an estimation of their uncertainty (The list of proton sites that were accounted for the calculation of these mean values for each temperature is available in Section 3 in the Supporting Information). Figure 8 (top) shows the evolution of $\operatorname{Ln}\left(k_{A B}\right)$ and $\operatorname{Ln}\left(k_{B A}\right)$ as a function of $1 / T$, for the standard and pure shift EXSY datasets.

The blue curves correspond to the linear regression that was applied to each dataset, showing for each approach a good correlation with the Arrhenius model on the considered temperature range. The error bars that have been determined for each point of these Arrhenius plots led us to discard the point determined at $290 \mathrm{~K}$ that is obviously on outlier for both approaches. The 2D spectra obtained at this temperature showed a degradation of the lineshape of the correlations, which suggests that a problem in the stability of the spectrometer occurred. Arrhenius curves were thus fitted to the 4 remaining points, and an estimate of the activation energies was extracted from these linear regressions. Again, a Monte Carlo based error analysis was carried out to evaluate the propagation of experimental uncertainty through the whole analytical workflow. The resulting distributions of the activation energies $E_{A B}$ and $E_{B A}$ are shown in Figure 8 (bottom). On the one hand, we remark that the mean values of the distributions generated by the standard and pure shift approaches are slightly different. The standard deviation of each energy distribution, which was found to increase significantly with the uncertainty of each data point, is smaller for the pure shift approach.

On the other hand, in both cases, the energy difference $E_{A B^{-}}$ $E_{B A}$ is of the order of $20 \mathrm{~kJ} \mathrm{~mol}^{-1}$, which is the same order of magnitude as what has been evaluated by DFT calculation for Trandolapril dissolved in $\mathrm{CDCl}_{3}{ }^{\left[{ }^{[2]}\right.}$ We note however that since the interconversion process is expected to be strongly depend- 
ent on all the factors that influence the solvation of this molecule, and notably the $\mathrm{pH}$ value, a more thorough study of the influence of the sample composition on this activation energy will be required in order to compare rigorously experimental and computational data.

\section{Conclusions}

In this paper, we report the implementation of the broadband homonuclear decoupling PSYCHE method in the NOESY pulse sequence for the purpose of recording pure shift EXSY spectra on the enzyme conversion inhibitor Trandolapril. The quality of the data obtained using this ultrahigh-resolution technique allows for studying the interconversion process of this molecule between two conformations, on a temperature range that is suitable for determining its activation energy. Notably, the artefacts arising from the reconstruction of the homonuclear decoupled free induction decay do not induce any significant degradation of the quality of the resulting pure shift EXSY spectra. The great simplification of the correlations lineshape that arises from the homonuclear decoupling allows for probing reliably a higher number of proton sites that would have remained out of reach using a standard EXSY pulse sequence.

These results pave the way for a rigorous analysis of the exchange process based on a comparison between ultra-high resolution EXSY data and energy values determined by a theoretical computation of this molecule modelled in the desired solvent. ${ }^{[6]}$ They also highlight the robustness of the pure shift EXSY experiment with regard to temperature variations, which shows that the performance of the homonuclear decoupling block remains acceptable when the time scale of the interconversion process changes.

Some limitations of the presented approach should however be reminded. First, the broadband homonuclear decoupling method implemented here has shown to be performant for exchange processes in the slow regime. As mentioned above, PSYCHE or Zangger-Sterk methods fail to produce efficient decoupling for exchange processes in the fast regime, i.e. yielding averaged resonances. Second, the main limitation to the implementation of such pure shift EXSY experiment is its lower sensitivity compared to standard pulse sequences, hence the longer experimental time that is needed to collect all the data. In the present work, pure shift EXSY spectra were recorded in $14 \mathrm{~h}$, whereas standard experiments only required $1 \mathrm{~h}$, which is due (i) to the lower sensitivity induced by the decoupling scheme, and (ii) to the time needed to accumulate all the increments of the pseudo-3D dataset. For simple spin systems with resolved sets of doubled resonances, it is obvious that standard methods should be used. Nevertheless, we argue that this approach becomes pertinent as soon as complex systems with crowded NMR spectra are investigated. This the case for instance for several biological samples, for which the required solvent, concentration or $\mathrm{pH}$ value lead to the observation of unresolved sets of doubled signals, which is a critical issue for standard approaches.
Finally, we note that different improvements can be made as regards implementation of pure shift methods in EXSY experiments. On the one hand, a number of interesting developments based either on the PSYCHE or the ZanggerSterk method have been introduced recently to remove artefacts from pure shift data, and notably overcome the general issue of strongly coupled protons. ${ }^{[37-42]}$ The SAPPHIRE method $^{[43]}$ has notably been shown to lead to a significant improvement of the quality of pure shift spectra by averaging to zero artefacts that arise from residual $J$ evolutions. It should be noted however that this latter method is time consuming because several scans should be acquired for each data point in order to perform the averaging process. The suitability of each approach for acquiring ultra-high resolution EXSY spectra in a reasonable amount of time should thus be addressed in the future. Notably, we have mentioned the approach consisting in implementing the pure shift element in the middle of the first evolution delay to improve the resolution along the indirect dimension of the resulting F1-PSYCHE-EXSY spectrum, which should also be evaluated regarding (1) the resolution that can be reached, and (2) the time needed for acquiring such experiment. On the other hand, the resolution enhancement that can be reached opens the way for a more accurate modelling of the evolution of the observed cross peaks, including chemical exchange and nOe transfers. Indeed, since a higher number of resolved correlations is available, it becomes possible to use more sophisticated relaxation models to fit data. The development of an analytical protocol to address reliably this question is currently underway in our group.

\section{Acknowledgements}

This work was supported by a scholarship from the University of Carthage-Tunisia. J.F. warmly thanks his partner Sandrine Bouchet for an unfailing assistance.

\section{Conflict of interest}

The authors declare no conflict of interest.

Keywords: chemical exchange - EXSY · NMR spectroscopy PSYCHE · pure shift

[1] D. R. P. Guay, Clin. Ther. 2003, 25, 713-775.

[2] L. Shen, J. L. Jiang, Y. H. Yu, Y. B. Wu, X. Y. Sun, Q. Q. Gu, Chem J Chinese U. 2012, 33, 153-157.

[3] A. D. Bain, Prog. Nucl. Magn. Reson. Spectrosc. 2003, 43, 63-103.

[4] I. R. Kleckner, M. P. Foster, Biochim. Biophys. Acta. 2011, 1814, 942-968.

[5] K. G. Orrell, Two-Dimensional Methods of Monitoring Exchange, Vol. (Eds.: R. K. Harris, R. L. Wasylishen), John Wiley \& Sons, Ltd, 2007.

[6] C. L. Perrin, T. J. Dwyer, Chem. Rev. 1990, 90, 935-967.

[7] J. Jeener, B. H. Meier, P. Bachmann, R. R. Ernst, J. Chem. Phys. 1979, 71, 4546-4553.

[8] A. G. Palmer, C. D. Kroenke, J. Patrick Loria, Nuclear Magnetic Resonance Methods for Quantifying Microsecond-to-Millisecond Motions in Biological Macromolecules, Vol. 339 (Eds.: T. L. James, V. Dötsch, U. Schmitz), Academic Press, 2001, pp.204-238. 
[9] G. Ivanova, B. Yakimova, S. Angelova, I. Stoineva, V. Enchev, J. Mol. Struct. 2010, 975, 330-334.

[10] A. A. Isab, M. I. M. Wazeer, Spectrochim Acta A Mol Biomol Spectrosc. 2006, 65, 191-195.

[11] A. Kálmán, F. Thunecke, R. Schmidt, P.W. Schiller, C. Horváth, J. Chromatogr. A. 1996, 729, 155-171.

[12] C. J. Falzone, P. E. Wright, S. J. Benkovic, Biochemistry. 1994, 33, 439442.

[13] J. E. H. Pucheta, D. Prim, J. M. Gillet, J. Farjon, ChemPhysChem. 2016, 17, 1034-1045.

[14] T. L. Religa, R. Sprangers, L. E. Kay, Science. 2010, 328, 98-102.

[15] J. Key, T. H. Scheuermann, P. C. Anderson, V. Daggett, K. H. Gardner, J. Am. Chem. Soc. 2009, 131, 17647-17654.

[16] J.-P. Demers, A. Mittermaier, J. Am. Chem. Soc. 2009, 131, 4355-4367.

[17] M. Doucleff, G. M. Clore, Proc. Natl. Acad. Sci. USA 2008, 105, 1387113876.

[18] R. Sprangers, A. Gribun, P. M. Hwang, W. A. Houry, L. E. Kay, Proc. Natl. Acad. Sci. USA 2005, 102, 16678-16683.

[19] Y. Nikolaev, K. Pervushin, J. Am. Chem. Soc. 2007, 129, 6461-6469.

[20] E. S. Kuloğlu, D. R. McCaslin, J. L. Markley, B. F. Volkman, J. Biol. Chem. 2002, 277, 17863-17870.

[21] P. Sarkar, C. Reichman, T. Saleh, R. B. Birge, C. G. Kalodimos, Mol. Cell. 2007, 25, 413-426.

[22] D. D. Boehr, H. J. Dyson, P. E. Wright, Chem. Rev. 2006, 106, 3055-3079.

[23] J. S. Fraser, M. W. Clarkson, S. C. Degnan, R. Erion, D. Kern, T. Alber, Nature. 2009, 462, 669.

[24] L. Kaltschnee, K. Knoll, V. Schmidts, R. W. Adams, M. Nilsson, G. A. Morris, C. M. Thiele, J. Magn. Reson. 2016, 271, 99-109.

[25] N. H. Meyer, K. Zangger, Angew. Chem. Int. Ed. 2013, 52, 7143-7146; Angew. Chem. 2013, 125, 7283-7286.

[26] P. Sakhaii, B. Haase, W. Bermel, R. Kerssebaum, G. E. Wagner, K. Zangger, J. Magn. Reson. 2013, 233, 92-95.

[27] J. Mauhart, S. Glanzer, P. Sakhaii, W. Bermel, K. Zangger, J. Magn. Reson. 2015, 259, 207-215.

[28] K. Zangger, Prog. Nucl. Magn. Reson. Spectrosc. 2015, 86-87, 1-20.
[29] K. Zangger, H. Sterk, J. Magn. Reson. 1997, 124, 486-489.

[30] J. A. Aguilar, S. Faulkner, M. Nilsson, G. A. Morris, Angew. Chem. Int. Ed. 2010, 49, 3901-3903; Angew. Chem. 2010, 122, 3993-3995.

[31] M. Foroozandeh, R. W. Adams, M. Nilsson, G. A. Morris, J. Am. Chem. Soc. 2014, 136, 11867-11869.

[32] M. Foroozandeh, R. W. Adams, M. Nilsson, G. A. Morris, J. Am. Chem. Soc 2014, 136, 11867-11869.

[33] M. Foroozandeh, R. W. Adams, N. J. Meharry, D. Jeannerat, M. Nilsson, G. A. Morris, Angew. Chem. Int. Ed. 2014, 53, 6990-6992; Angew. Chem. 2014, 126, 7110-7112.

[34] R. Wagner, S. Berger, J. Magn. Reson. Ser. A 1996, 123, 119-121.

[35] D. Pitoux, Z. Hu, B. Plainchont, D. Merlet, J. Farjon, D. Bonnaffé, N. Giraud, Magn. Reson. Chem. 2018, 56, 954-962.

[36] M. Foroozandeh, G. A. Morris, M. Nilsson, Chem. Eur. J. 2018, 24, 1398814000.

[37] S. Tassoti, P. Novak, C. P. Butts, K. Zangger, ChemPhysChem. 2018, 19, 3166-3170.

[38] S. K. Mishra, N. Suryaprakash, Magn. Reson. Chem. 2018, 56, 893-909.

[39] V. M. R. Kakita, J. Bharatam, Magn. Reson. Chem. 2018, 56, 963-968.

[40] J. Ilgen, L. Kaltschnee, C. M. Thiele, J. Magn. Reson. 2018, 286, 18-29.

[41] B. Görling, W. Bermel, S. Bräse, B. Luy, Magn. Reson. Chem. 2018, 56, 1006-1020.

[42] I.E. Ndukwe, A. Shchukina, V. Zorin, C. Cobas, K. Kazimierczuk, C. P. Butts, ChemPhysChem. 2017, 18, 2081-2087.

[43] P. Moutzouri, Y. Chen, M. Foroozandeh, P. Kiraly, A. R. Phillips, S. R. Coombes, M. Nilsson, G. A. Morris, Chem. Commun. 2017, 53, 1018810191.

Manuscript received: March 11, 2019

Revised manuscript received: April 25, 2019

Accepted manuscript online: April 29, 2019

Version of record online: 


\section{ARTICLES}

Simplify 2D EXSY: We show that pure shift methods can be implemented to simplify crowded 2D EXSY spectra, hereby paving the way to an ultra-high-resolution analysis of chemical exchange for processes in the slow regime timescale. The robustness of this approach is demonstrated using the PSYCHE method on a pharmaceutical compound undergoing slow conformational exchange. The resolution enhancement achieved on a broad range of temperatures ( 40 Kelvin) allows for determining exchange rates and activation energies of this interconversion process on several proton sites.

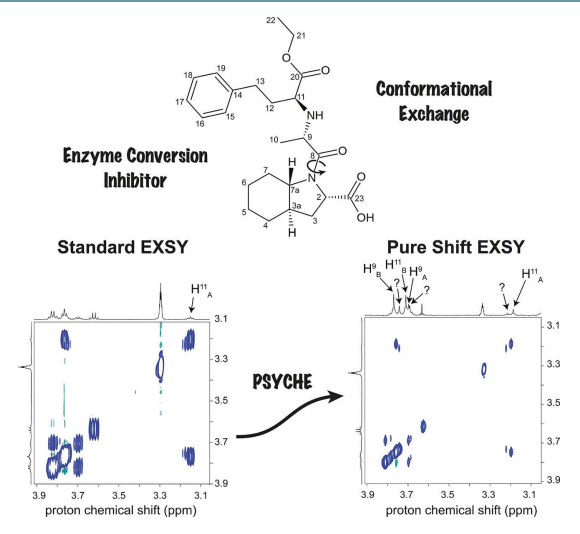

G. Aloui, S. Bouabdallah, J. P. Baltaze, J. E. H. Pucheta, S. Touil, J. Farjon, N. Giraud* $^{*}$

$1-10$

Monitoring Conformational Changes in an Enzyme Conversion Inhibitor Using Pure Shift Exchange NMR Spectroscopy

\section{\#\# SPACE RESERVED FOR IMAGE AND LINK}

Share your work on social media! ChemPhysChem has added Twitter as a means to promote your article. Twitter is an online microblogging service that enables its users to send and read short messages and media, known as tweets. Please check the pre-written tweet in the galley proofs for accuracy. If you, your team, or institution have a Twitter account, please include its handle @username. Please use hashtags only for the most important keywords, such as \#catalysis, \#nanoparticles, or \#proteindesign. The ToC picture and a link to your article will be added automatically, so the tweet text must not exceed 250 characters. This tweet will be posted on the journal's Twitter account (follow us @ChemPhysChem) upon publication of your article in its final (possibly unpaginated) form. We recommend you to re-tweet it to alert more researchers about your publication, or to point it out to your institution's social media team.

\section{ORCID (Open Researcher and Contributor ID)}

Please check that the ORCID identifiers listed below are correct. We encourage all authors to provide an ORCID identifier for each coauthor. ORCID is a registry that provides researchers with a unique digital identifier. Some funding agencies recommend or even require the inclusion of ORCID IDs in all published articles, and authors should consult their funding agency guidelines for details. Registration is easy and free; for further information, see http://orcid.org/.

\section{J. E. H. Pucheta}

N. Giraud http://orcid.org/0000-0003-2322-339X

S. Touil

S. Bouabdallah

J. Farjon

G. Aloui

J. P. Baltaze 